\title{
Statistical geometric affinity in human brain electric activity
}

\author{
A. Chornet-Lurbe \\ Servicio de Neurofisiología Clínica, Hospital Arnau de Vilanova, 46015-València, Spain \\ J. A. Oteo* \\ Departament de Física Teòrica, Universitat de València, 46100-Burjassot, València, Spain
}

$$
\text { J. } \operatorname{Ros}^{\dagger}
$$

Departament de Física Teòrica and Instituto de Física Corpuscular, Universitat de València, 46100-Burjassot, València, Spain

(Received 10 April 2006; revised manuscript received 29 November 2006; published 29 May 2007)

\begin{abstract}
The representation of the human electroencephalogram (EEG) records by neurophysiologists demands standardized time-amplitude scales for their correct conventional interpretation. In a suite of graphical experiments involving scaling affine transformations we have been able to convert electroencephalogram samples corresponding to any particular sleep phase and relaxed wakefulness into each other. We propound a statistical explanation for that finding in terms of data collapse. As a sequel, we determine characteristic time and amplitude scales and outline a possible physical interpretation. An analysis for characteristic times based on lacunarity is also carried out as well as a study of the synchrony between left and right EEG channels.
\end{abstract}

DOI: 10.1103/PhysRevE.75.051918

PACS number(s): 87.19.La, 05.45.Tp

\section{INTRODUCTION}

The human electroencephalogram (EEG) is the collective by-product of the electric activity of the brain. Nowadays, electroencephalography constitutes a widely used noninvasive clinical technique since a lot of wave forms associated with normal and abnormal physiological states have been classified.

From the physical point of view, the EEG measures the potential difference between electrodes positioned according to international standards as a function of time. Each of these integrates the activity of the cortical neurons in the neighborhood of its contact surface which, in turn, is the result of countless discharge cascades across the network of neurons located in deeper areas of the brain. Due to this intricate origin one could think of the EEG as an approximately random time series. At the same time, however, neural electrical signals also carry important physiological information. This would suggest some short term structure and regularity. Furthermore [1], there is a number of geometric and biophysical constraints on brain wiring which impinge on the way pulses combine to form the EEG. The final situation would likely be an intricate balance between these two limiting aspects of the EEG, namely, regularity and randomness. The complexity of EEG records emerges as the outcome of that interplay.

A field in which electroencephalography has played a particularly significant role is sleep studies [2]. One distinguishes between rapid-eye-movement (REM) sleep and nonREM (NREM) sleep. In turn in NREM sleep one differentiates between light sleep, with stages I and II, and slow sleep, with stages III and IV or just with no distinction between them (stage III/IV). Relaxed (closed eyes) wakefulness possesses its own features and is also included in sleep

\footnotetext{
*Electronic address: oteo@uv.es

†Electronic address: rosj@uv.es
}

analysis. Throughout the night sleep phases alternate, at intervals of unpredictable duration, following patterns which vary according to the age or the presence or absence of cerebral pathology. The practical definition of these stages comes primarily from the analysis of EEG wave forms according to standard Rechtschaffen-Kales (RK) rules [3] that attend mainly to rhythms and amplitudes, although further physiological signals are also considered. Together they constitute the so-called sleep polysomnography.

Clinicians are trained to identify different wave forms in EEGs as signatures of particular states of the brain. Furthermore, they are required to automate as much as possible their responses, and for the sake of convenience the data are displayed in a fully standardized way in a time-amplitude plane. Convention has lead the medical clinicians to some results which, as far as we know, have not yet been fully explained in a consistent mathematical way from the EEG raw data. It is the aim of this paper to advance such an analysis for the following features.

(1) Convertibility of the EEG samples of the different sleep stages and relaxed wakefulness into each other. As described in Sec. III this is achieved by appropriately adjusting the polygraph scales and certainly deserves an explanation. In Sec. V we propound a statistical explanation for that finding and argue that it constitutes strong evidence for the existence of universal traits in the brain electric activity during sleep.

(2) Detection of rhythms by assignment of frequencies to the EEG signals. This is usually done in daily practice by determination of the zero-crossing rate (ZCR) of the electric potential values. This procedure is not free from ambiguities and so other possibilities of defining instantaneous frequencies are worth considering. In Sec. IV we explore a proposal in that direction, based on Hilbert transform, which is afterwards fully analyzed in Sec. V.

(3) The striking similarity between REM sleep and wakefulness is well-known to neurophysiologists. In some respects REM and NREM sleep are more different from a 
medical point of view. It would then be interesting to extract, from the raw data, numerical indicators echoing this distinctive situation. In Sec. V we introduce a proper time parameter which reflects that condition, while in Sec. VI we numerically show that in other aspects REM is nearer to other genuine sleep stages.

(4) Clinicians have identified a certain hierarchy among the different sleep stages based mainly on qualitative details. By a convenient analysis of the data we turn this sleep phase ordering quantitative in Sec. V. A certain degree of hierarchy of sleep stages is also indicated by the Hilbert phase synchrony between left and right EEG channels as we point out in Sec. VII.

In Sec. II we describe the data on which we base our conclusions. In attaining our results the use of the Gabor analytic signal method [4,5] based on Hilbert transform [6] applied to the EEG time-series has been instrumental. We have also exploited the idea of lacunarity [7], a concept borrowed from fractal geometry.

Universality in brain electrical activity has already been reported in the literature: different aspects of spatial universality have been considered for awake subjects [8] and for various cognitive tasks [9]. Alternatively, the heartbeat during sleep has been shown to follow universal patterns for healthy individuals [10]. In our case the alleged EEG universality is based on the data collapse of appropriate probability density functions (PDF) into master curves. This rescaling is a hallmark of universality in physiological sleep and unveils an unexpected property hidden in the RK filing rules. It buttresses the existence of a common cerebral mechanism with a few tunable regimes and goes one step beyond the complexity reduction performed by the sleep phases classification. Our results suggest also some more speculative interpretation which we defer to the discussion in Sec. VIII.

\section{DATA DESCRIPTION}

The time series to be analyzed were sampled from raw data obtained with a digital polygraph Alice 3, v.1.19 from Healthdyne Technologies, Inc. Usual parameters were recorded in addition to EEG: electrocardiogram, thorax and abdomen breathing movements, nose-mouth air flux, eye movements, chin and legs muscle contractions, body position, arterial saturation of oxygen, and snore. The EEG was recorded by means of two pairs of electrodes C3-A1 and C4-A2 on the scalp, according to the international system $10 / 20$. Each channel captures the signal from the corresponding hemisphere in the central area albeit they do not represent the activity of the whole hemisphere. The EEG signal was steadily filtered during the whole process (0.55 Hz high pass, $45 \mathrm{~Hz}$ low pass). Polysomnographies were $7 \mathrm{~h}$ long and the sampling rate was $100 \mathrm{~Hz}$.

All the data we consider in our analysis were taken in routine observations of patients coming to the hospital for diagnosis in the Sleep Unit. Sample selection was carried out according to the following criteria. First, absence of artifacts was required, namely no apparent interference with signals (biological or otherwise) not originated in brain. Second, samples were fully representative of wakefulness in a relaxed

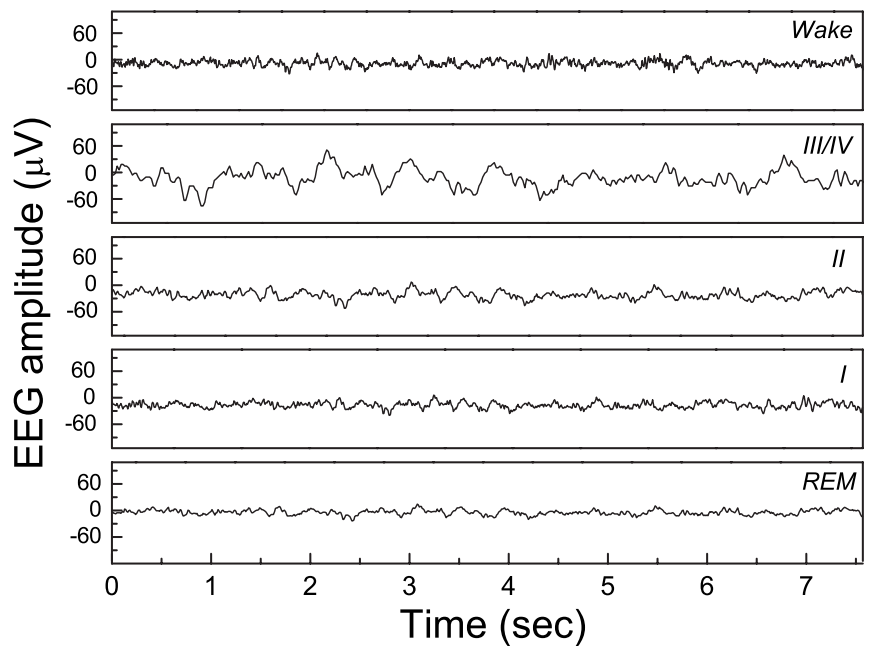

FIG. 1. EEG sample of REM sleep phase (bottom) and synthetic replicas from it which mimic the remaining stages.

state and different sleep phases. They fit, with no ambiguity, the general criteria for sleep phase reading, commonly used in clinical practice, following the time honored Rechtschaffen-Kales (RK) standardized sleep manual [3].

As a last requirement, selected samples of a particular phase and subject were neither adjoining nor clustered in time. They originated at well-separated time intervals during the night. Each selected time series consists of 3000 points which correspond to $30 \mathrm{~s}$ epochs. The whole data-basis consists of 23 subjects. Every subject contributes with six samples per wakefulness-sleep phase and per channel, with a few exceptions where less than six samples were recorded. The total number of samples is 1368 .

As customary in clinical practice the time-amplitude representation of the data followed the convention: $5-10 \mu \mathrm{V} / \mathrm{mm}$ pen deflection (amplitudes axis) and $1.5-3 \mathrm{~cm} / \mathrm{s}$ paper speed (time axis) which amount to epochs of either 10 or $20 \mathrm{~s}$. EEG snapshots are displayed on monitors with horizontal resolution of at least 1400 pixels in the data display area.

\section{GRAPHICAL EXPERIMENTS WITH DATA}

The generalized use of digital polygraphs has allowed further data treatment. In particular by appropriate readjustments of the axes one can change the appearance of any given stage records and make them look like representatives of any other stage.

We can describe this result in geometric terms. Taken at face value an EEG record, $u(t)$, amounts to a set of points in the time-amplitude plane and we pose the ensuing question: can we change the appearance of an EEG sample by a scaling affine transformation in such a way that it may be classified as belonging to another different stage? Here the term affine transformation alludes to the fact that the scaling factors may be different in each axis, time, and amplitude.

Figure 1 illustrates the result of the aforementioned graphical experiment. The graph at the bottom corresponds to a true REM record of about $7 \mathrm{~s}$ long. The remaining four 


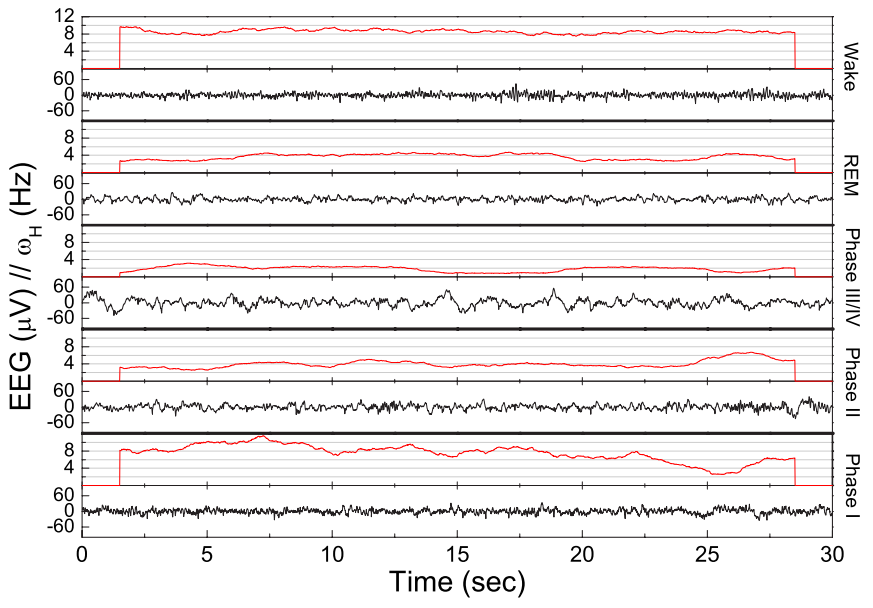

FIG. 2. (Color online) Raw epochs of five EEG stages amplitudes $u(t)$, lower subpanels (black lines) together with their corresponding smoothed Hilbert instantaneous frequencies $\langle\omega(t)\rangle$, upper subpanels (red lines).

graphs are labeled I, II, II/IV, and Wake because that is what standard application of clinical reading says they are, at least as far as EEG records are concerned. The key point is that they are synthetic electroencephalograms obtained through the appropriate affine transformation of the real raw REM record in the bottom panel. Certainly, the synthetic EEGs cannot exhaust the whole panoply of details used for clinical identification of stages. Thus some particular short length wave forms (spindles or $k$-complexes) are not generated by this procedure, but the main traits are well-reproduced. This can be assessed by direct comparison of Fig. 1 with Fig. 2 in which epochs of a true EEG are shown in the lower subpanels of every state.

This phenomenon is preserved when running samples are considered if we allow for some occasional fine-tuning. The main drawback is the lack of systematics of the procedure. We propose an approach based on Hilbert transform to elucidate the mutual transformation of stages into one another through a statistical analysis of the corresponding PDF.

In the authors' opinion this interconversion of sleep phases reveals some deep and distinctive structure in sleep EEGs. It would be interesting to ascertain whether other sets of physiological signals share this interchangeability among their elements. Numeric experiments with synthetic signals such as different types of colored noise and the logistic map in chaotic regimes with various parameter values show that this kind of interconversion is not a general feature.

\section{THE ANALYTIC EEG}

The EEG time series described above are far from being periodic and there is no natural way of defining instantaneous frequencies. Generally speaking, one can find in the literature different proposals for assigning an instantaneous frequency to a nonperiodic signal $[11,12]$. The conventional detection of rhythms by neurophysiologists goes through a supple zero-crossing bookkeeping procedure along some time interval (this is the familiar ZCR of signal treatment in other fields) Thus the number of times the EEG signal crosses the zero potential axis per time unit is at the base of the very definition of EEG frequency used in neurophysiology [13] which, in practice, is applied with further considerations. For instance, very brief zero crossings might be ignored in this counting. This and other subtleties render the method a little bit fuzzy with respect to an efficient mathematical implementation.

We shall instead resort to the concept of analytic signal $[4,5]$, based on Hilbert transform [6], which is widely used in theory of signal, circuits, and systems. Closer to the present issue, the analytic signal concept has been applied in [14] to study chaotic synchronization in the context of nonlinear dynamics.

Given a function $u(t)$ for which the Fourier transform exists, then the so-called analytic signal is defined as

$$
\Psi(t)=u(t)+i v(t)
$$

where $v(t)$ is the Hilbert transform (HT) of $u(t)$. The HT of a one-variable scalar function $u(t)$ is defined by

$$
v(t) \equiv \frac{1}{\pi} \mathcal{P} \int_{-\infty}^{\infty} \frac{u(x)}{t-x} d x
$$

where $\mathcal{P}$ stands for Cauchy principal value of the integral. Thus the function $\Psi(t)$ in Eq. (1) is analytic in the sense of Cauchy-Riemann.

In the present case, $u(t)$ will represent the EEG signal and Eq. (1) states that $u(t)$ is the real part of an analytic function. We can write down that equation in polar form

$$
\Psi(t) \equiv A(t) e^{i \phi(t)}
$$

where $A$ and $\phi$ stand for the Hilbert amplitude an phase, respectively. The definition of an instantaneous frequency readily follows

$$
\omega(t) \equiv \frac{d}{d t} \phi(t)=\frac{u(t) v^{\prime}(t)-u^{\prime}(t) v(t)}{u^{2}(t)+v^{2}(t)}
$$

where the prime stands for time derivative.

The definition (2) is not much of a practical application when dealing with experimentally sampled data. A practical recipe [6] may, however, be established from the observation that the HT of the functions $\sin t$ and $\cos t$ are known to be $-\cos t$ and $\sin t$, respectively.

Let $\left\{u_{0}, u_{1}, u_{2}, \ldots, u_{n-1}\right\}$ stand for the sampled data values $u\left(t_{k}\right)=u_{k}$. For the sake of simplicity, let us suppose that samples are equally spaced in time by an interval $\Delta t$ and that $n$ is even. This time series may be represented as the real Fourier sum

$$
u_{F}(t)=\sum_{k=0}^{n / 2-1}\left[a_{k} \cos (2 \pi k t / T)+b_{k} \sin (2 \pi k t / T)\right] \text {, }
$$

where $T=n \Delta t$. The HT of $u_{F}$ reads then 


$$
v_{F}(t)=\sum_{k=0}^{n / 2-1}\left[a_{k} \sin (2 \pi k t / T)-b_{k} \cos (2 \pi k t / T)\right] .
$$

The determination of the $n$ Fourier coefficients is carried out by solving the linear algebraic system defined by the conditions $\left\{u_{F}\left(t_{k}\right)=u_{k}\right\}$, with $k=0, \ldots, n-1$. A discrete fast Fourier transform routine [15] may be used to this end.

Substitution of Eqs. (5) and (6) into Eq. (4) provides us with an explicit formula to calculate the Hilbert instantaneous frequency. The derivatives in Eq. (4) are obtained from Eqs. (5) and (6).

Notice that, according to Eq. (4), $\omega$ may occasionally take negative values. This outcome is viewed as a serious drawback in engineering applications literature [11]. Accordingly, the input signals are narrow-band filtered in order to avoid their emergence. There is nothing mathematically incorrect with negative frequencies, not even from a physical interpretation point of view.

Because of Eq. (2) every value of the instantaneous frequency $\omega(t)$ provided by the analytic signal gets contributions from the entire epoch. We tend to think of this feature as a virtue of the method since all the data in the time series, which share the clinical filing, contribute to some extent to define every value of the instantaneous frequency. In contrast, we must mention that the same argument has been put forward in the literature as a formal drawback, the objection being about causality violation $[16,17]$.

\section{EEG DATA MINING}

The aim of the data mining presented here is threefold. First of all, we show that the analytical signal ansatz provides a reasonably good description of the rhythms observed in clinical practice when contrasted with local averages $\langle\omega(t)\rangle$ taken over some time interval. This observation buttresses the appropriateness of treating the EEG signal as a complex variable.

Second, we show that both raw Hilbert frequencies and amplitudes gather into a well-defined PDF for every wakefulness-sleep phase. In Sec. I we invoked Fig. 1 as a strong indication that affine transformations in the timeamplitude plane achieve the desired stage interchangeability. From now on we replace the EEG signal description in terms of $u(t)$ with $A(t)$ and $\omega(t)$. These two magnitudes are closer to the RK rules and lead eventually to a systematics since their values yield well-defined histograms for wakefulness and sleep stages. From them we extract probability density functions which we denote by $g_{k}(A)$ and $f_{k}(\omega)$, respectively, where $k$ stands for the stage index $(k=\mathrm{I}$, II, III/IV, REM, Wake). We designate by $\left\{\bar{A}_{k}, \sigma_{k}^{A}\right\},\left\{\bar{\omega}_{k}, \sigma_{k}^{\omega}\right\}$ their respective means and standard deviations.

Third, we show that with the appropriate choice of scaling and shifting parameters one gets

$$
\alpha_{k} f_{k}\left[\left(\omega-\bar{\omega}_{k}\right) / \alpha_{k}\right]=F(\omega),
$$

$$
\beta_{k} g_{k}\left[\left(A-\bar{A}_{k}\right) / \beta_{k}\right]=G(A),
$$

where $F$ and $G$ exhibit net data collapse. The numbers $\alpha_{k}$ and $\beta_{k}$ have no dimension. When $F$ and $G$ have unit standard deviation, as in the present case, they then take the same values as $\sigma_{k}^{\omega}$ and $\sigma_{k}^{A}$, respectively.

We interpret the rescaling between PDFs provided by the previous equations as the replica, in the new amplitudefrequency description, of the EEG congruency procedure defined in the amplitude-time plane. Due to the instrumental role played by the PDF in this interpretation we call this property statistical affinity. As a by-product, we carry out a determination of proper frequencies and amplitudes for relaxed (closed eyes) wakefulness and every sleep phase, since the PDFs are not scale free.

\section{A. Adequacy of Hilbert frequencies to the definition of EEG rhythms}

The time series of Hilbert frequencies $\omega(t)$ are as irregular as their EEG counterpart. As aforementioned, instantaneous frequencies can even assume negative values. However, EEG filing does not need such an intensive determination of instantaneous frequencies as that provided by $\omega(t)$. Figure 2 presents epochs of $30 \mathrm{~s}$ of true EEG samples corresponding to the classes we study. In parallel to these EEG snapshots, we have plotted the locally averaged determination of values $\langle\omega(t)\rangle$. The time interval chosen for adjacent data points averaging is $3 \mathrm{~s}$, albeit this value is not crucial at all. The interest of Fig. 2 stems from the fact that it witnesses that $\langle\omega(t)\rangle$ essentially agrees to the clinical determination of rhythms from EEG snapshots. This feature is general across the collection of samples we have analyzed.

We consider that these results establish a successful nexus between the mathematical procedure and the visual analysis. Notice that the determination of the instantaneous Hilbert frequency is based on the assumption of the analytic signal principle with no a priori connection with the ZCR.

A further perspective about the connection between the clinical and mathematical procedures is the following. In Fig. 3 we represent the PDF of the smoothed instantaneous frequencies obtained from the whole set of EEG samples. The rhythm intervals allowed by the RK rules [3] are also indicated in the same Fig. 3 by labeled horizontal boxes. We observe a remarkable intersection of both types of information albeit not complete. Taking into account that some short length wave forms (spindles, $k$-complexes, ...) are not considered in our analysis, the agreement is acceptable.

Fourier power spectrum analysis is a common tool when dealing with stationary time-series. EEGs are not stationary since the computation of statistics as the mean amplitude is not necessarily time-invariant. Notwithstanding that fact, we have computed the Fourier power spectrum for every EEG sample and normalized it to unit power. Figure 4 represents the average power spectra of wake-sleep phase states in log$\log$ scales. Lines of slope corresponding to $1 / f$ and $1 / f^{2}$ noise are given for visual reference. The peak at about $9 \mathrm{~Hz}$ in the wakefulness power spectrum corresponds to the presence of $\alpha$-complexes charateristics of relaxed closed-eyes 


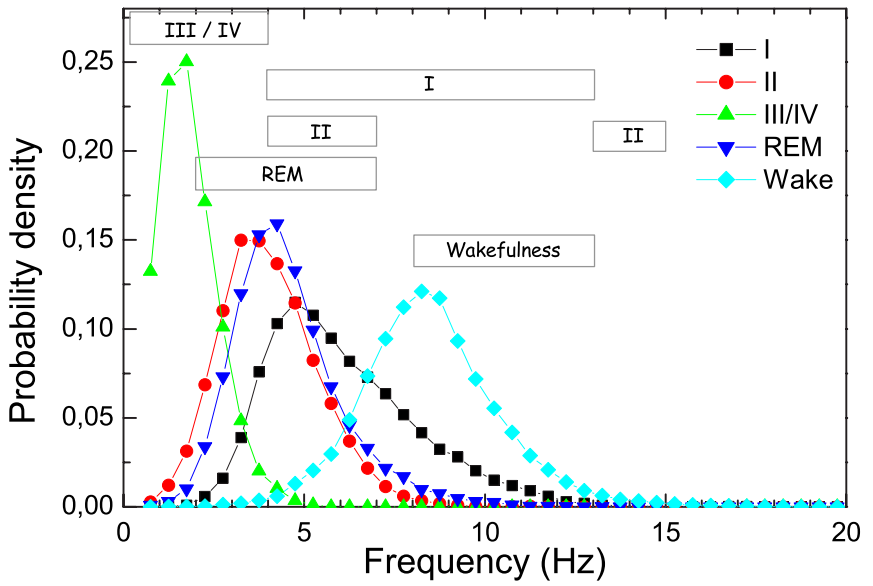

FIG. 3. (Color online) Smoothed Hilbert frequency PDF for wakefulness and sleep phases obtained from the whole set of EEG samples. Horizontal boxes stand for the intervals where EEG frequencies are to lie according to the RK protocol.

wakefulness EEGs. The peak at about $10.5 \mathrm{~Hz}$ in the phase II power spectrum corresponds to spindles (which are characteristic wave forms lasting about $1 \mathrm{~s}$ ). Obviously, the height of both peaks depends on the whole $\alpha$-complex and spindles content of the sample set. It is a common practice to seek power-law behavior in power spectra but due to the limited frequency range in our data we do not proceed in that direction.

\section{B. Statistics for Hilbert frequencies}

Once a positive visual link between the experimental outcomes and $\langle\omega(t)\rangle$ has been established, we study the statistics of raw Hilbert frequencies. To this end we commence by producing an approximate PDF from normalized statistical histograms. This computation is the analog of that for the smoothed frequencies in the last part of Sec. V A. The new outcomes are shown in Fig. 5 on a vertical log-scale. The

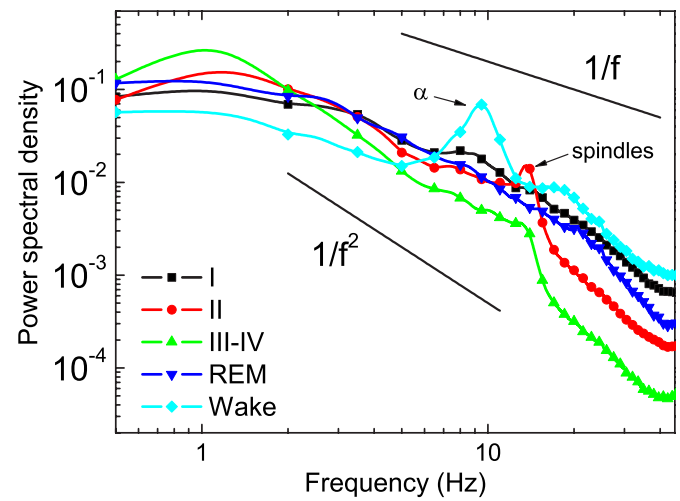

FIG. 4. (Color online) Normalized average Fourier power spectra for wake-sleep phases obtained from the whole set of EEG samples. The slopes for $1 / f$ and $1 / f^{2}$ noise are also indicated. The peak at about $9 \mathrm{~Hz}$ in wakefulness power spectrum corresponds to $\alpha$-complexes. The peak at about $10.5 \mathrm{~Hz}$ in phase II corresponds to the presence of spindles.

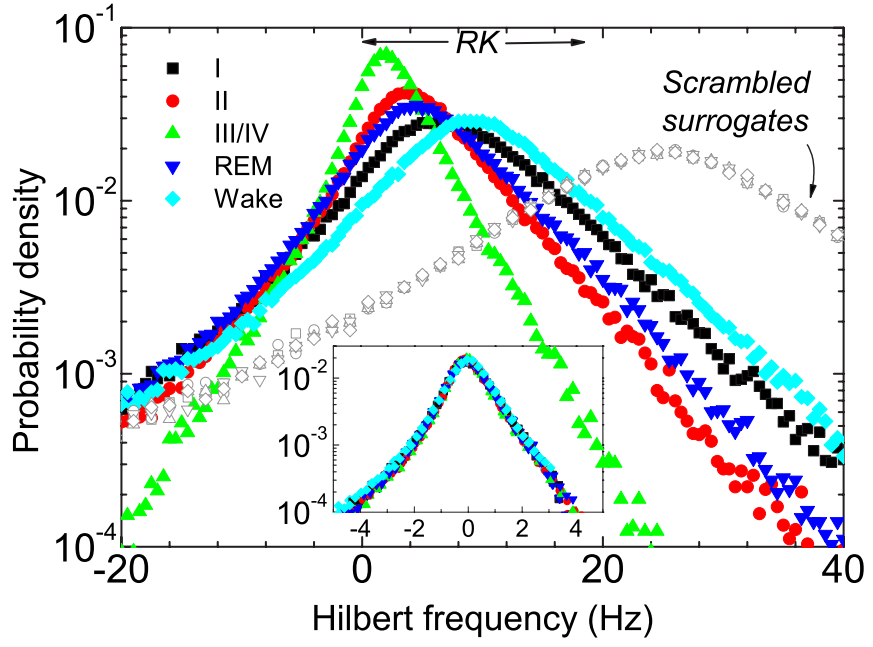

FIG. 5. (Color online) Raw Hilbert frequency PDF for wakefulness and sleep phases. The PDF for scrambled surrogates are explicitly indicated as well as the frequency range used by RK filing rules. The inset shows the data collapse into a master curve after rescaling of the five EEG PDFs.

profiles are not Gaussian because they exhibit longer tails. The overall frequency range used in the polysomnographic analysis is explicitly marked and labeled as RK in the plot. It is worth noticing that only rhythms inside this interval intervene in the sleep phase classification procedure. Instead, well-defined profiles extend outside the RK interval.

The statistical modes, i.e., the most frequent values, for $\omega$ accommodate well in the RK frequency bands of the EEG background of relaxed wakefulness and sleep phases.

According to Fig. 5, in first approximation the density profiles are symmetric about some shift frequency $\bar{\omega}_{k}$ and go as $\exp \left[-\left|\omega-\bar{\omega}_{k}\right| / \Omega_{k}\right],(k=\mathrm{I}$, II, III/IV, REM, Wake), where $\Omega_{k}$ stands for a decay constant to be interpreted as a characteristic frequency of the stage. A linear fit to the log of the PDF tail yields an estimate of the proper time for every stage: $\tau_{k} \equiv 2 \pi / \Omega_{k}$. These results are given in Table I and we propose in Sec. VIII a possible meaning for them. Furthermore, the deeper the sleep the longer the proper time of the stage such as is witnessed in Table I. Error bars have been estimated through bootstrap statistical method [18] and correspond to one standard deviation. One-thousand replicas among the 23 subjects were produced for every wake-sleep phase to this end. No significant bias was detected in the distributions of the proper times bootstrapped values.

Figure 5 contains also the outcome of the analysis when the amplitudes in the raw EEG records were previously scrambled. Those are the so-called scrambled surrogate data and the situation corresponds to pose the null hypothesis: EEGs are random time series. To obtain the surrogate data we generate, for every sample, a random permutation $\pi$ and transform the time series $\left\{u_{i}\right\}$ into $\left\{\bar{u}_{i}=u_{\pi(i)}\right\}$, where $\pi(i)$ is the image of index $i$ under $\pi$. The fact that the curve is not flat is due to the finiteness of the sampling rate and the $45 \mathrm{~Hz}$ filtering of the signal. The slope of this distribution yields a $0.65 \mathrm{~s}$ characteristic time. Figure 5 shows that some essential rhythms are definitely lost after EEG amplitude scrambling. Thus the null hypothesis is not true, and the EEGs are not random time series. 
TABLE I. EEG statistics from the PDFs in Figs. 5 and 6.

\begin{tabular}{lcccccc}
\hline \hline State & $\bar{\omega}$ & $\begin{array}{c}\sigma_{\omega} \\
(\mathrm{Hz})\end{array}$ & $\begin{array}{c}\bar{A} \\
(\mu \mathrm{V})\end{array}$ & $\begin{array}{c}\sigma_{A} \\
(\mu \mathrm{V})\end{array}$ & $\begin{array}{c}\tau \\
(\mathrm{s})\end{array}$ & $\begin{array}{c}a \\
(\mu \mathrm{V})\end{array}$ \\
\hline I & 6.9 & 10.4 & 9.2 & 5.9 & $0.93 \pm 0.04$ & $5.7 \pm 0.3$ \\
II & 4.8 & 7.8 & 13.1 & 9.7 & $1.21 \pm 0.04$ & $8.9 \pm 0.3$ \\
III/IV & 2.5 & 4.6 & 20.6 & 13.8 & $1.82 \pm 0.05$ & $12.7 \pm 0.7$ \\
REM & 5.3 & 9.0 & 8.5 & 5.2 & $1.04 \pm 0.02$ & $4.4 \pm 0.2$ \\
Wake & 9.6 & 11.3 & 9.3 & 5.9 & $0.92 \pm 0.04$ & $5.1 \pm 0.6$ \\
\hline \hline
\end{tabular}

The similarity among the five profiles in Fig. 5 suggests their possible renormalization into a master curve. Indeed, when the five probability density profiles $f_{k}(\omega)$ are shifted and rescaled so as to have vanishing average and unit standard deviation, according to Eq. (7), then a net data collapse into a unique master curve is observed (see inset of Fig. 5). It points toward a common underlying mechanism driven essentially by two tuning parameters: $\bar{\omega}$ and $\sigma^{\omega}$, that may take five pairs of values $\left\{\bar{\omega}_{k}, \sigma_{k}^{\omega}\right\}$. Under this perspective, a sleep stage change corresponds to a sudden variation of those parameter values, as regards the EEG rhythm.

\section{Statistics for Hilbert amplitudes}

The analysis with respect to EEG Hilbert amplitudes proceeds similarly to the statistics of frequencies above. In Fig. 6 we plot the different PDF for the analytic signal amplitudes in every stage. Scrambled surrogates produce the same PDFs as raw data.

The inset of Fig. 6 shows the data collapse after renormalization of Hilbert amplitude distributions, $g_{k}(A),(k=\mathrm{I}$, II, III/IV, REM, Wake), according to Eq. (8). As in the frequencies case, we think of a unique underlying mechanism driven by two parameters, mean $\bar{A}$ and standard deviation $\sigma^{A}$, whose values are determined by the physiological state. Furthermore, we carry out a determination of the characteristic

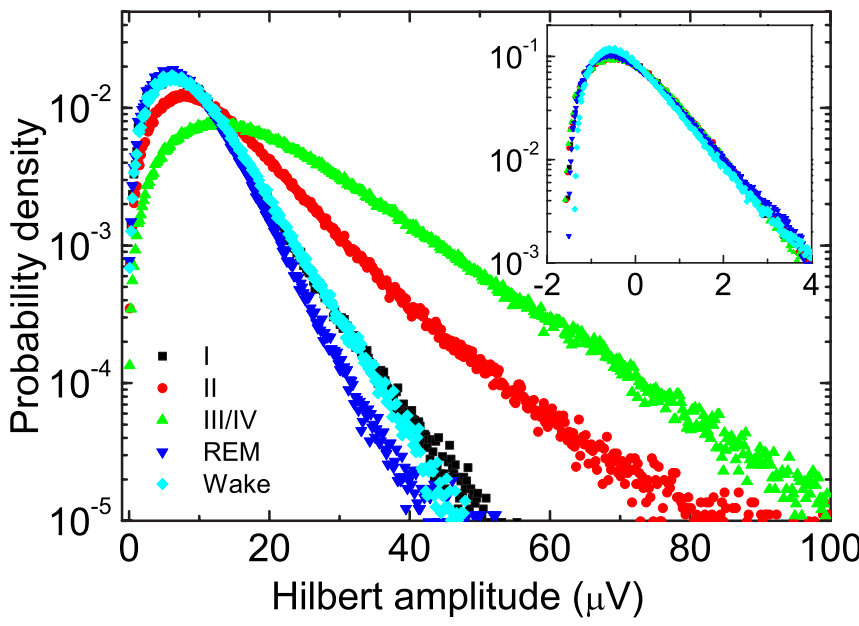

FIG. 6. (Color online) Analytic signal amplitude PDF for wakefulness and sleep phases. The inset corresponds to data collapse into a master curve after rescaling of the PDF. amplitude of every stage. A linear fit to the log of the distribution tail yields an estimate of the proper amplitude $a_{k}$ for every stage. These results are reported in Table I. Error bars have been estimated through bootstrap [18] following the same scheme used above in the analysis of Hilbert frequencies. No significant bias was detected in the distributions of the proper amplitudes bootstrapped values.

Certainly, the raw EEG amplitudes could be directly used, without further ado, to perform an equivalent analysis since, unlike frequencies, there is no ambiguity concerning their definition. We have checked the corresponding outcomes, which produce well-defined PDFs, roughly symmetric with respect to zero amplitude, that exhibit exponential tails too. For the sake of consistency with our scheme based on the analytic signal hypothesis we choose to deal with Hilbert amplitudes $A(t)$.

\section{CHARACTERISTIC TIMES FROM LACUNARITY}

The proper time introduced in Sec. V B can be compared with other characteristic times associated with the wakesleep stages by a different way of analyzing the temporal texture of EEG time series. For this purpose the concept of lacunarity proves to be helpful. It complements the information furnished by the notion of fractal dimension since objects with different degrees of homogeneity in its structure may share the same value of fractal dimension. Originally introduced in fractal geometry $[7,19,20]$, it may actually be applied to classify a time or space pattern whether fractal or not $[21,22]$ characterizing the degree of homogeneity. It has met utility in a wide range of fields such as astrophysics [23], fluid mechanics [24], ecology [25], or medicine [26], at least at a descriptive level. Here we will endow the procedure with a recipe to determine characteristic times in the pattern.

Following [21] we define lacunarity according to the socalled gliding-box algorithm. Suppose a time series is given containing $N$ equally spaced data $\left\{u_{i}\right\}_{i=1}^{N}$, with vanishing mean. We generate a binary time series by assigning the mass value $1(0)$ to positive (negative or vanishing) values of $u_{i}$. This binary time series is then scanned with a onedimensional window of length $r$ which glides on it one point at a time. At every position of the control segment (gliding box) the mass inside is evaluated. The collection of gliding boxes allows us to define the distribution of mass $n(M, r)$ as the number of gliding boxes with length $r$ and mass $M$. Dividing $n(M, r)$ by the total number of boxes it results in a probability function $Q(M, r)$. When the mass in a gliding box 
is independent of its position then we have a translational invariant time series. Of course, translational invariance may depend on the time scale.

Lacunarity at scale $r$ is defined as

$$
\Lambda(r)=\frac{Z^{(2)}(r)}{\left[Z^{(1)}(r)\right]^{2}},
$$

where $Z^{(n)}, n=1,2$, are the statistical moments associated with $Q$,

$$
Z^{(n)}(r) \equiv \sum_{M} M^{n} Q(M, r) .
$$

In the case of translational invariant time series $Z^{(2)}(r)$ $=\left[Z^{(1)}(r)\right]^{2}$, hence $\Lambda(r)=1$. Time series with lacunae of all sizes will have $\Lambda(r) \gg 1$ whereas patterns with some degree of regularity, for instance, with single-sized lacunae, are expected to have low $\Lambda(r) \geqslant 1$. Lacunarity is to be considered as a scale-dependent measure of texture.

Plotnick et al. [22] have carefully discussed the meaning of the shape of $\Lambda(r)$ profiles. Lacunarity for a time series of length $N$, at the two extreme values $r=1$ and $N$ is $\Lambda(1)$ $=1 / P$, where $P$ stands for the fraction of occupied sites, and $\Lambda(N)=1$. For other $r$ values the shape of $\Lambda(r)$ depends of the nature of the signal. A random time series from a uniform distribution gives rise to a concave upwards $\Lambda(r)$ because holes of any size are present at any scale. Lacunarity decreases steadily when increasing the length of the box because it becomes more translationally invariant. No change of scale emerges. The analytic shape of this profile is readily computed as the probability for a gliding window of length $r$ to contain mass $M$ is given by the binomial probability distribution

$$
Q(M, r)=\frac{1}{2^{r}}\left(\begin{array}{c}
r \\
M
\end{array}\right),
$$

and therefore Eq. (9) reduces to

$$
\Lambda(r)=1+\frac{1}{r}
$$

for a random pattern. A regular pattern such as a square wave of wavelength $\lambda$ gives rise to a concave downwards $\Lambda(r)$ with a high constant value at small enough box lengths. Then its value suddenly decays to $\Lambda=1$ after the box size exceeds $\lambda$ because translational invariance is reached. The transition corresponds to the proper regularity scale of the pattern. A striking situation in between is observed when pulses of duration $\lambda$ are randomly generated. A transition from downward to upward concave shape at about $r=\lambda$ appears. This witnesses a change in the pattern. Thus looking for such downward-upward crossovers in $\Lambda(r)$ profiles is a tool to extract characteristic time scales from irregular time series. Notwithstanding this, the quantitative determination of the transition point is not straightforward, as we explain below.

To use lacunarity in the study of EEG signals [27] we first subtract the global average and then convert them into binary symbolic time series (positive versus negative potential state). To make possible an unbiased comparison among the

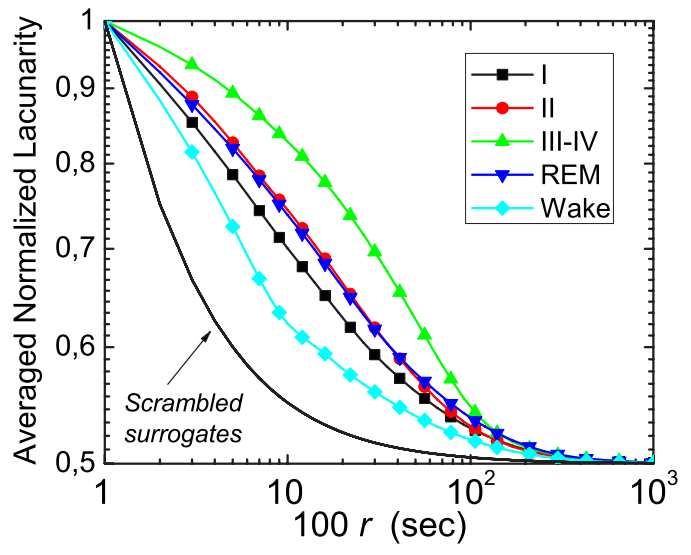

FIG. 7. (Color online) Average normalized lacunarity profiles, see Eq. (12), for the different wake-sleep states as a function of the gliding box size $r$ in log-log scales. The solid concave upward curve corresponds to scrambled surrogates.

profiles $\Lambda(r)$ from the various EEG samples we propose the following variant. The whole task is performed twice. The first [second] time, 1's are assigned to positive [negative] values of the time series and a profile $\Lambda^{+}(r)\left[\Lambda^{-}(r)\right]$ is obtained. As a numeric zero is rare in the time series, no bias is expected whether it is assigned either as positive or negative. Furthermore, the ratio between the number of positive and negative values in a time series differs usually from unity, thus we work with $\Lambda_{H}$, half the harmonic mean:

$$
\frac{1}{\Lambda_{H}}=\frac{1}{\Lambda^{+}}+\frac{1}{\Lambda^{-}}
$$

This expression for the lacunarity is normalized at the origin: $\Lambda_{H}(1)=1$, a feature very interesting in order to build up average lacunarity profiles for the different groups of wakesleep states, which we plot in Fig. 7. For the sake of comparison we include the normalized lacunarity of scrambled EEGs (solid line, no symbol). We observe that wakefulness lacunarity is the closest profile to a pure random process. As far as sleep gets deeper the lacunarity profile moves away, phase I (or drowsiness) first and then phase II, and reaches maximum regularity at phase III-IV. Note the huge similarity between REM sleep and phase II. By contrast, we remind that the EEG of the former is known to resemble a wake state in other respects; and more importantly, it becomes clear from Fig. 7 that there are transitions from convex to concave shape as the size of the gliding-box increases. We note in passing that for a self-affine time series, $\Lambda_{H}$ is a power law and then would appear as a straight line in Fig. 7.

The quantitative estimation of the point where the transit occurs is not simple. The transit may even take place along an interval where $\log \left[\Lambda_{H}(\log r)\right]$ is quasilinear and thus the inflexion point is not well-defined in practice. As a matter of fact, the systematic numerical determination of downwardupward crossovers in lacunarity profiles has not been considered in the literature. We found that their conventional location by the vanishing of the second derivative turns out to exhibit, in the present case, numerical instabilities. Closely 


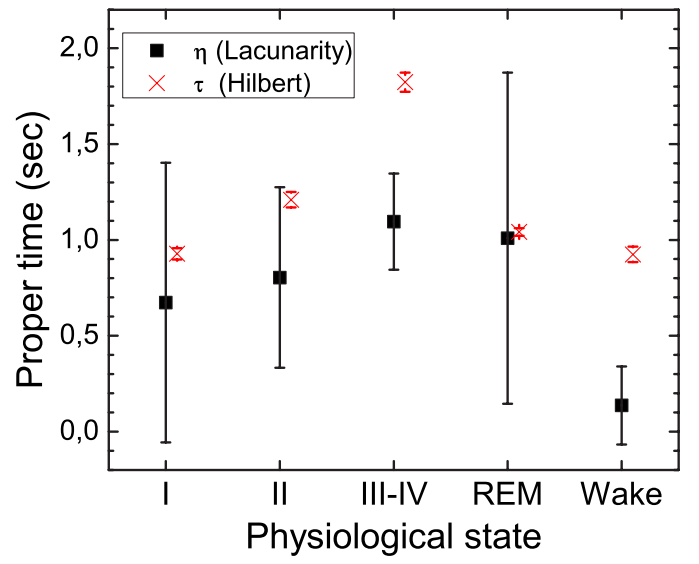

FIG. 8. (Color online) Characteristic times $\eta$ and $\tau$ as a function of the wake-sleep phase. Values of $\eta$ are obtained from maximal curvature of $\log \left(\Lambda_{H}\right)$ and then averaged over EEG samples. Values of $\tau$ are obtained from slopes in the Hilbert frequency PDF (see Table I). Error bars refer to one standard deviation.

related to the second derivative of a function $h(x)$ is its local curvature $\rho(x)$,

$$
\rho(x)=\frac{d^{2} h(x) / d x^{2}}{\left\{1+[d h(x) / d x]^{2}\right\}^{3 / 2}} .
$$

So, we have chosen to locate the transition at the point $r$ $=\eta$ of maximal curvature of the lacunarity profiles in log-log representation. This furnishes more stable results.

To locate the point $\eta$ from the discrete unevenly sampled function $h(\log r)=\log \Lambda_{H}(\log r)$ we have used a gliding box of size $2 N+1$ and fitted a parabola (the simplest analytic form yielding second derivatives). Stable results were obtained for $N \geqslant 3$. The values shown correspond to $N=3$. Computation of the location of this point for every EEG sample yields the statistic that we plot in Fig. 8. Notice that both lacunarity and Hilbert methods yield a similar physiological hierarchy for the values $\eta$ and $\tau$. Error bars correspond to one standard deviation.

In [22] a variant to the computation of the lacunarity is proposed. It is only applicable to the time series of positive terms and avoids the binary reduction of the data allowing the analysis of quantitative data. The idea is to compute lacunarity using the sum of the distribution in the gliding box, which is interpreted as beginning the analysis at a coarser level of resolution. We have carried out this type of analysis looking for characteristic times in $A(t)$, since they are positive. Lacunarity profiles in this scheme are not normalized at the origin any longer. Thus, unlike the former analysis, we cannot obtain average lacunarity profiles. The statistics of characteristic times obtained from all EEG samples with this variant presents a dispersion at least twice that of the binary version. This is likely due to the more irregular structure of these lacunarity profiles so that the search strategy for a maximal curvature point in $\log \Lambda$ might not be the most adequate. Due to these drawbacks we choose not to abound with this variant.

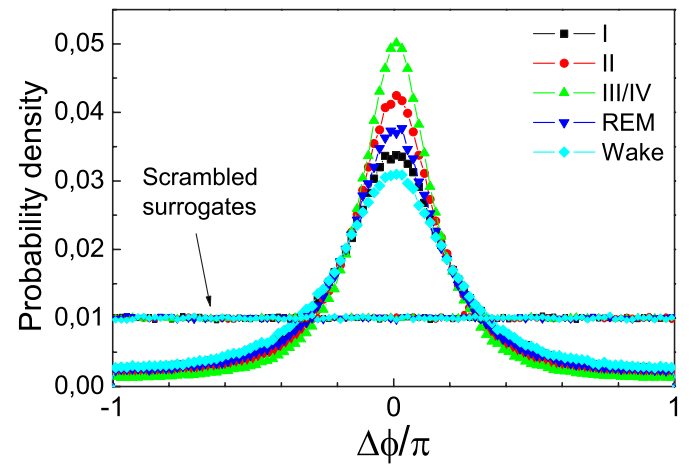

FIG. 9. (Color online) PDF for the left-right Hilbert phase differences of the wake-sleep stages.

\section{PHASE SYNCHRONY BETWEEN EEG CHANNELS}

Throughout our analysis we have left aside the Hilbert phase $\phi$ in Eq. (3). As we have already noted in passing, there is presently an increasing interest about synchrony phenomena in complex systems. In this section we gather some results concerning synchrony of left-right EEG channels, via the Hilbert phase.

Some studies that handle long enough time series track the time evolution of the phases of the system and may identify phenomena such as resonances or phase locking. We are not in position of carrying out such a type of analysis as the samples in our data set are not long enough for that purpose. We can instead study phase synchrony in a statistical sense.

In Fig. 9 we plot the normalized histograms obtained for every wake-sleep state of the Hilbert phase difference $\Delta \phi(t)$ between left and right channels. $\Delta \phi(t)$ was mapped in the interval $[-\pi, \pi]$. For scrambled amplitude EEGs, the probability density is uniform. For wake-sleep stages, once again, there is a hierarchy in the degree of synchrony. Thus, as far as sleep gets a deeper state, phase synchrony between leftright EEG channels is steadily enhanced, in a statistical sense. Notice that REM sleep ranks between phases I and II rather than next to wakefulness. This shows once more that in some aspects REM sleep is more similar to wakefulness while in other it appears clearly among the sleep stages.

\section{DISCUSSION}

The present work has been motivated by the observation that EEG samples representative of wakefulness and sleep phases can be transformed into each other by affine transformations.

First, we gain insight about the phenomenon directly on the time-amplitude plane. Next, in order to deal with a systematic procedure, the principle of the analytic signal turned out to be of utility to define instantaneous frequencies. These frequencies, averaged over a short time interval, result in good agreement with clinical criteria. After that, the frequency-amplitude description has been developed in statistical terms.

The PDF for amplitudes and raw frequencies we have built up from a large set of EEG samples are not scale free. As a matter of fact, their exponential tails have led us to 
determine proper times (or frequencies) and amplitudes. If we consider the pairs of characteristic times and amplitudes $\left\{\tau_{k}, a_{k}\right\}$ in Table I then, with the global perspective, it becomes evident that slow sleep $\tau_{k}$ correlates with the largest amplitudes $a_{k}(k=\mathrm{II}, \mathrm{III} / \mathrm{IV})$. Correspondingly, wakefulness, drowsiness, and REM sleep share the faster rhythms and the smaller amplitudes. This feature is familiar to neurophysiologists, albeit no quantification attempt had been done to date, to the best of our knowledge.

Furthermore, with the exception of REM sleep, the product $\sigma_{k}^{A} \sigma_{k}^{\omega}=67 \pm 6 \mu \mathrm{V} \mathrm{Hz}$, in a way that brings to mind uncertainty relations. Although we do not assign any deep significance to this fact, it is anyway curious because here amplitude and frequency are not Fourier transform duals of one another.

It could be interesting to extend the study to other physiopathological situations such as epileptic seizures, anesthesia, and coma which comprise, as a matter of fact, a broad variety of degrees or stages and where proper time-amplitude correlations seem to be prevented.

The experimental situation with sleep polysomnographies has offered a rather exceptional starting point for the present analysis. We mean that we are given, $a b$ initio, a number of well-defined sets of physiological states. Their classification is the outcome of observational work and their current definition seems not to have historically followed a straightforward course [28]. Furthermore, as the guidelines of that scheme have nothing to do with any mathematical formalism, it is striking that the statistics on amplitudes and, particularly, Hilbert frequencies obey well-defined patterns over broad intervals. A fortunate enough fact that has led us to point out the existence of data collapse for PDFs.

We have carried out an independent determination of sleep-wake EEG characteristic times from lacunarity profiles. To this end, we introduced a normalized lacunarity and identified the concave-convex transition by the points of maximal curvature of the profiles. We hope this procedure may also be used with other types of binary time series. The characteristic times obtained this way, although slightly different from those given by Hilbert frequencies, still preserve the physiological hierarchy among wake-sleep stages (Fig. $8)$.

In summary, we have clarified from a quantitative point of view some features observed rather qualitatively in clinical practice [see items (1)-(4) in the Introduction]. We close this section with some, certainly more speculative, comments which we consider worth doing.
It is presently accepted that physiological information travels through the nervous system coded in wave trains of different duration [30]. The EEG is the integration of a huge number of wave trains traveling to the cortex. We think of proper times $\left\{\tau_{k}\right\}$ as being a sort of global measure of the average duration of the EEG wave forms in every stage. From their values we conclude that neuronal information during deep sleep is coded in longer wave trains, perhaps needed for the recovery function commonly accepted for it.

Instead, shorter wave trains during wakefulness and drowsiness are consistent with a higher number of simultaneous brain tasks. Dreams, thought to happen during REM sleep, convey some degree of mental activity (we remind the term paradoxical sleep). Arguably, the short values for $\tau_{R E M}$ and $\eta$ we have found render the REM sleep closer to a wakefulness stage than to deep sleep. REM sleep may not have a recovery function, which is a generally accepted fact.

Characteristic EEG amplitudes $\left\{a_{k}\right\}$ are to be interpreted as a measure of the amount of neuron population that synchronize their activity, at least as the phenomenon is recorded on the scalp. Large EEG amplitudes, such as in sleep phase III/IV, can be attained only under some degree of collective synchrony. Phase synchrony between EEG channels points in this direction.

Finally we interpret the data collapse for the PDF as a signature of the presence of a common underlying mechanism. Hence the (sudden) transitions from any wakefulnesssleep stage to another would correspond to discrete changes of the tuning parameter values. The nature of that mechanism, as well as the proper times and amplitudes, should be universal in the sense that it emerges from data from a variety of subjects. Although the situation might appear favorable, we do not propound here to explain the transit phenomenon as a phase transition in the statistical mechanics sense, as this should require further analysis [29]. For that purpose one should study the very EEG samples when the wakefulness-sleep phase transit occurs. Notice that the EEG samples we have selected for analysis were especially chosen to be bona fide representatives of the stages and not of the transitions. Indeed, further work in this direction seems worth doing.

\section{ACKNOWLEDGMENTS}

We thank A. Fernández-Soto for illustrative discussions. This work has been partially supported by contracts MCyT/ FEDER, Spain (Grant No. FIS2004-0912) and Generalitat Valenciana, Spain (Grant No. ACOMP06/042).
[1] S. B. Laughlin and T. J. Sejnowski, Science 301, 1870 (2003).

[2] J. A. Hobson, Nature (London) 437, 1254 (2005).

[3] A. Rechtschaffen and A. Kales, A Manual of Standardized Terminology, Techniques and Scoring System for Sleep Stages of Human Subjects, Public Health Service, NIH Publication No. 204 (US Government Printing Office, Washington, DC, 1968).

[4] D. Gabor, J. Inst. Electr. Eng., Part 3 93, 429 (1946).
[5] J. Ville, Cables Transm. 2A, 61 (1948).

[6] S. L. Hahn, in The Transforms and Applications Handbook, edited by A. D. Poularikas (IEEE, New York, 2000).

[7] B. B. Mandelbrot, The Fractal Geometry of Nature (Freeman, San Francisco, 1977).

[8] P. Seba, Phys. Rev. Lett. 91, 198104 (2003).

[9] J. Bhattacharya and H. Petsche, Proc. R. Soc. London, Ser. B 
268, 2423 (2001).

[10] P. Ch. Ivanov et al., Nature (London) 383, 323 (1996).

[11] B. Boashash, Proc. IEEE 80, 519 (1992).

[12] B. Boashash, Proc. IEEE 80, 539 (1992).

[13] E. Niedermeyer and F. Lopes da Silva, Electroencephalography. Basic Principles, Clinical Applications and Related Fields (Lippincott, Williams \& Wilkins, New York, 1998).

[14] M. G. Rosenblum, A. S. Pikovsky, and J. Kurths, Phys. Rev. Lett. 76, 1804 (1996).

[15] W. H. Press et al., Numerical Recipes in Fortran: The Art of Scientific Computing (Cambridge University Press, Cambridge, England, 1997)

[16] M. S. Gupta, Am. J. Phys. 43, 1087 (1975).

[17] L. Mandel, Am. J. Phys. 42, 840 (1974).

[18] B. Efron and R. J. Tibshirani, Stat. Sci. 1, 54 (1986).

[19] B. B. Mandelbrot, Gaussian Self-affinity and Fractals: Globality, the Earth, $1 / f$ Noise and R/S (Springer, New York, 2002).

[20] Y. Gefen, B. B. Mandelbrot, and A. Aharony, Phys. Rev. Lett. 45, 855 (1980).
[21] C. Allain and M. Cloitre, Phys. Rev. A 44, 3552 (1991).

[22] R. E. Plotnick, R. H. Gardner, W. W. Hargrove, K. Prestegaard, and M. Perlmutter, Phys. Rev. E 53, 5461 (1996).

[23] I. Stern, in ADASS VI ASP Conference Series, Vol. 125, edited by G. Hunt and H. E. Payne (Astronomical Society of the Pacific, San Francisco, 1997).

[24] L. A. Smith, J. D. Fourier, and E. A. Spiegel, Phys. Lett. A 114, 465 (1986).

[25] E. M. Rauch, M. M. Millonas, and D. R. Chialvo, Phys. Lett. A 207, 185 (1995).

[26] A. J. Einstein, H. S. Wu, and J. Gil, Phys. Rev. Lett. 80, 397 (1998).

[27] A. Chornet-Lurbe, J. A. Oteo, and J. Ros, Rev. Neurol. 35, 415 (2002).

[28] M. Hirshkowitz, Sleep Medicine Rev. 4, 169 (2000).

[29] X. Campi and H. Krivine, Phys. Rev. C 72, 057602 (2005).

[30] W. J. Freeman, Int. J. Bifurcation Chaos Appl. Sci. Eng. 2, 451 (1992). 